\title{
Avances recientes en dermatología comunitaria 2016-2017. Teledermatología, un modelo de enseñanza y asistencia en atención primaria a la salud
}

\author{
María de Guadalupe Chávez-López¹, Guadalupe E. Estrada-Chávez², Maribel Orozco-Figueroa³, \\ Adriana Solís-Rivera ${ }^{4}$, Jessy Solchaga-Rosas ${ }^{5}$, Felipe Armendariz-Valle ${ }^{6}$ y Roberto A. Estrada-Castañón ${ }^{7 *}$ \\ ${ }^{1}$ Servicio de Dermatología, Hospital General de Acapulco, Secretaría de Salud; ${ }^{2}$ Secretaría de Dermatología Comunitaria A.C., Facultad de Medicina, \\ Universidad Autónoma de Guerrero; ${ }^{3}$ Educación Médica e Investigación en Salud; ${ }^{5}$ Departamento de Capacitación en Salud; ${ }^{6}$ Ciencias en Epidemiología \\ - Colegio de Bachilleres del Estado de Guerrero; ${ }^{7}$ Presidente de Dermatología Comunitaria México, Subdirección de Enseñanza e Investigación. México
}

\section{Resumen}

Introducción: La dermatología comunitaria (DC) está integrada por dermatólogos, epidemiólogos y personal afín. Tiene 27 años de existencia en el Estado de Guerrero. Por condiciones de inseguridad se cambió el modelo presencial de enseñanza y atención dermatológica a la forma virtual, a través de teledermatología. Objetivo: Determinar la utilidad de la capacitación y enseñanza virtual a través de la telemedicina en DC. Método: Estudio transversal, descriptivo, observacional y analítico, en el que se evaluaron los resultados de la enseñanza-aprendizaje en el personal de salud capacitado por la red de telemedicina mediante nueve cursos que llegaron a 35 centros de telemedicina en el Estado de Guerrero. Resultados: Se capacitó en dermatología básica a 2465 trabajadores de la salud, evaluando sus conocimientos antes y después de cada curso. Se validó estadísticamente la utilidad de esta modalidad de enseñanza con una muestra al azar de 611 asistentes, con resultados satisfactorios. Discusión: La enseñanza a través de la red de telemedicina representa un avance en DC al llegar a un mayor número de asistentes en varios centros de recepción en forma simultánea, minimizando los gastos de transporte y salvaguardando la integridad de los participantes, con la ventaja de dar continuidad a los propósitos de $D C$.

PALABRAS CLAVE: Telemedicina. Teledermatología. Epidemiología cutánea. Comunidades marginadas. Dermatología comunitaria.

\section{Abstract}

Introduction: Community dermatology $(C D)$ is integrated by dermatologists, epidemiologists and interested people. It has 27 years of experience at the Guerrero State. Due to unsafety issues, the teaching and medical attention model had to be changed from presential to virtual trough telemedicine. Method: Transversal, descriptive, observational and analytic study, in which results from teaching-learning of the health personal that was capacitated throughout the telemedicine network at the Guerrero State were evaluated, during nine courses that reached 35 telemedicine centers. Results: 2465 health workers were capacitated in basic dermatology, which were evaluated in their general knowledge of dermatology before and after every course. The utility of this modality of teaching was statistically evaluated with a random simple in 611 attendees with satisfactory results. Discussion: Teaching through telemedicine network, represents an improvement for $C D$ by reaching a higher number of attendees to several reception centers simultaneously, minimizing transportation expenses and improving the safety of the participants at the activities, with the advantage of allowing continuity of the CD purposes.

KEY WORDS: Telemedicine. Teledermatology. Cutaneous epidemiology. Marginated communities. Community dermatology.

Correspondencia:

${ }^{*}$ Roberto A. Estrada-Castañón

Juan Sebastián Elcano, 222-312

Fracc. Hornos

Fecha de recepción: 18-08-2018

C.P. 39355, Acapulco, Gro., México

Fecha de aceptación: 20-08-2018

Gaceta Med Mex. 2018;154(Suppl 2): S36-S40

E-mail: restrada_13@hotmail.com

DOI: 10.24875/GMM.18004576

Disponible en PubMed www.gacetamedicademexico.com 


\section{Introducción}

La dermatología comunitaria (DC) es un programa que tiene como propósito fundamental llevar los beneficios de esta especialidad a los grupos marginados que carecen de servicios médicos especializados. Para cumplir con este objetivo, se capacita al personal del primer nivel de atención de la salud de áreas remotas en la identificación y el manejo elemental de las dermatosis comunes ${ }^{1}$. La experiencia en el área epidemiológica ha permitido tener un conocimiento de la patología prevalente en cada región del Estado ${ }^{2,3} \mathrm{y}$ establecer un programa de enseñanza basado en las 10 dermatosis más frecuentes. Otro objetivo primordial es la identificación de pacientes que evolucionan sin control por carecer de personal capacitado para identificarlos, los que se canalizan, cuando así se requiere, a un segundo o tercer nivel.

En su evolución, el programa DC ha atravesado por diferentes etapas. En la primera, del año 1990 al año 2000 , es cuando se crea el concepto de $D^{4}$ y se consolida la investigación de la patología dermatológica prevalente en el Estado de Guerrero a través del sistema de "sitios centinela" ${ }^{5}$ del Centro de Investigación de Enfermedades Tropicales. En la segunda etapa, del año 2000 al año 2014, se forma la Asociación Civil que lleva su nombre, se hacen convenios con la Secretaría de Salud y el Desarrollo Integral para la Familia (DIF) Estatal, encargados del aspecto logístico y DC de los cursos y jornadas para capacitar al personal de salud del primer nivel de atención en salud en las siete jurisdicciones estatales. El trabajo se estructuró en cada jornada con los asistentes al curso para mostrarles, en la práctica, el manejo de las dermatosis; asimismo, se contó con estudiantes de medicina de la Universidad Autónoma de Guerrero, residentes de dermatología de diferentes instituciones (Instituto Nacional de Pediatría, Hospital General de México, Hospital General Dr. Manuel Gea González) y dermatólogos invitados, tanto del país como extranjeros, miembros de la American Academy of Dermatology $(A A D)$ y de la Fundación Internacional para la Dermatología, coordinados por el personal de nuestra asociación (Figura 1). Durante la jornada dermatológica, un aspecto relevante fue la distribución en forma gratuita de los medicamentos necesarios. La experiencia de este periodo se vierte en comunicaciones científicas internacionales, que consolidan y expanden el concepto de $D C,{ }^{6-8}$ y se enriquece con trabajos

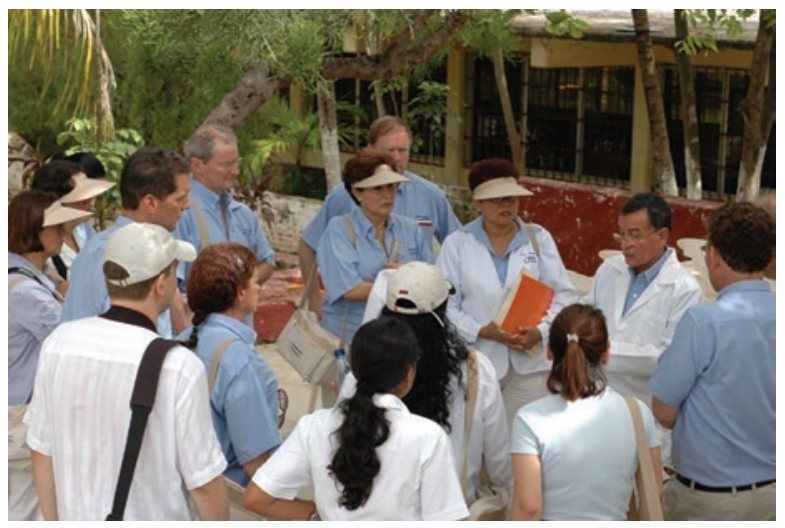

Figura 1. Integrantes de la Jornada Dermatológica en Copala, Guerrero, 2005.

similares de otros países. ${ }^{9,10}$ Este esquema de atención y enseñanza presenciales abarcó mas de una década. Las condiciones de inseguridad se agudizaron en el país, lo que nos obligó a disminuir y finalmente suspender las visitas comunitarias, al hacer crisis en nuestra entidad en 2014 (Conflicto de Ayotzinapa).

Debido a la necesidad de atención dermatológica en la población de escasos recursos, decidimos continuar con el programa de DC iniciando una tercera etapa, fundamentada en los avances tecnológicos de las comunicaciones, ${ }^{11}$ a través de la red de telemedicina de la Secretaría de Salud en el Estado de Guerrero. Se implementaron los cursos de teledermatología y se continuó asesorando por teleconsulta a las áreas más apartadas de nuestra entidad. En 2010 se llevó a cabo el primer curso de teledermatología en Chilpancingo, Guerrero, con el apoyo y la asesoría de médicos de la AAD (Figura 2). En sus inicios, las consultas se efectuaron mediante teléfonos celulares, sin un registro sistematizado.

A partir de 2016, la DC toma un giro diferente al utilizar los recursos tecnológicos de información y comunicación, y organiza a través de la Red de Telemedicina de la Secretaría de Salud los cursos de manera virtual mediante los módulos que están distribuidos en el Estado de Guerrero y en otros Estados, lo que nos permitió expandirnos a Chiapas y Oaxaca, bajo la consideración de que el sureste de nuestro país comparte las condiciones de marginación y pobreza más acentuadas. Otro de los enfoques de importancia para la DC son las jornadas de investigación, con un propósito específico de captación de pacientes nuevos o recuperación de los que han abandonado el tratamiento. 


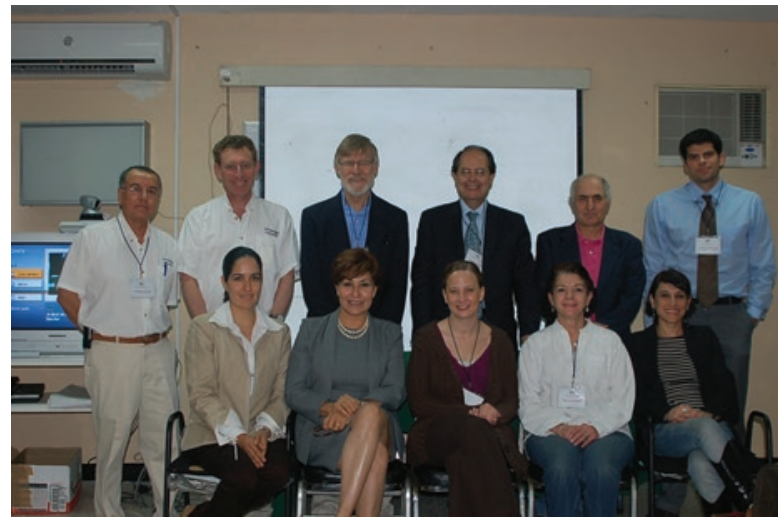

Figura 2. Primer Curso de Teledermatología con asesoría de miembros de la American Academy of Dermatology.

El objetivo de este trabajo es determinar la utilidad de la capacitación y la enseñanza virtual a través de la telemedicina en la DC.

\section{Método}

Se realizó un estudio transversal, descriptivo, observacional y analítico, en una muestra del personal de salud que se capacitó e instruyó por la Red de Telemedicina en el Estado de Guerrero durante los años 2016 y 2017. Se tomó una muestra aleatoria de 611 personas capacitadas en 19 jurisdicciones de salud para determinar la utilidad de la capacitación y la enseñanza a distancia. Se practicó un examen de dermatología básica previo y otro posterior al curso para evaluar su nivel de conocimientos. Se hizo un registro de las teleconsultas mediante un formato de historia clínica de cada paciente, enviado por el personal capacitado en los cursos, para asesorar sus diagnósticos y orientar el tratamiento.

\section{Resultados}

En 2016 y 2017 se impartieron ocho cursos en 35 sedes de las siete regiones del Estado de Guerrero (Acapulco, Costa Grande, Costa Chica, Zona Centro, Norte, Tierra Caliente y Montaña), a una población de 2465 trabajadores de la salud repartidos en cuatro categorías: 922 médicos generales (37.1\%), 77 pasantes (3.5\%), 769 enfermeras (31.2\%) y otras categorías que incluyeron especialistas, administrativos, promotores y técnicos en atención primaria de salud $(28.2 \%)$ (Figura 3$)$.

La muestra para validar estadísticamente la utilidad de la enseñanza por teledermatología arrojó resultados del $95 \%$ de confianza (hipótesis de la media $Z=4.40384151)$. Los cursos generaron 143 teleconsultas, que se agrupan en cinco categorías: dermatosis de origen infeccioso (47; 16.3\%), discromías (23; $1.6 \%)$, dermatosis inflamatorias $(43 ; 3.0 \%)$, tumores cutáneos $(12 ; 0.83 \%)$, genodermatosis $(6 ; 0.4 \%)$ y miscelánea (12; 0.83\%) (Figura 4).

\section{Discusión}

La enseñanza a través de la red de telemedicina representa un avance en DC al llegar a un mayor número de asistentes en varios centros de recepción en forma simultánea, minimizando los gastos de transporte y salvaguardando la integridad de los participantes, con la ventaja de dar continuidad a los propósitos de la DC. La forma presencial, que se realizó hasta los inicios de la presente década, tenía una cobertura de enseñanza anual aproximada de 400 trabajadores de la salud, a los que se proporcionaban los cursos en la ciudad más importante cercana a su área de trabajo. Este número contrasta marcadamente con las cifras alcanzadas por teledermatología (2465 personas capacitadas). Por otra parte, el promedio de consultas, entre 100 y 150 proporcionadas en las visitas comunitarias, disminuyó marcadamente en la nueva modalidad, ya que son suplidas por la asesoría constante a través de la red de telemedicina, para casos complicados o que no se han podido resolver en el ámbito local. Sin menospreciar el papel que otras categorías de trabajadores del área de la salud tienen en la atención de las áreas remotas, las enfermeras constituyen en grupo importante, como se señala en la Figura 3, no solo por su número, sino también porque al formar parte de la comunidad con frecuencia son las principales representantes de los servicios de salud. ${ }^{12}$ Para proporcionar atención médica a las áreas más aisladas, los servicios de salud en Guerrero han implementado las «unidades móviles", integradas por un grupo heterogéneo de trabajadores de la salud, a las que se dedica un curso de dermatología al año.

La categorización de las enfermedades consultadas se muestran en la Figura 4, y difiere en forma importante de lo comunicado por Lepe, et al..$^{13}$ en cuanto a la clasificación de las dermatosis, porque en nuestra casuística nos basamos en las enfermedades que vemos con mayor frecuencia, entre las que predominan las infecciosas, seguidas por las dermatosis inflamatorias. Los casos que lo ameritan, por su 


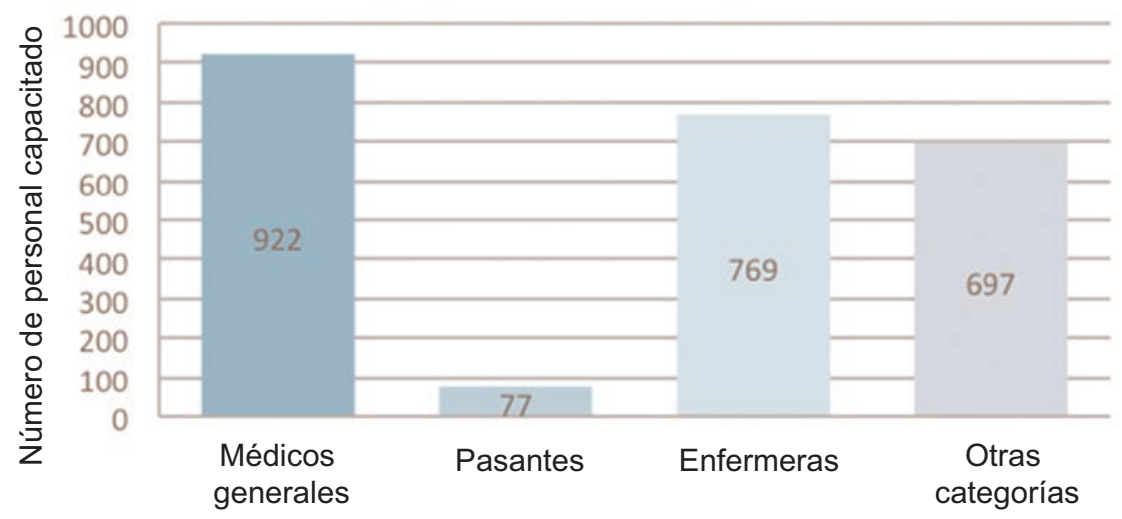

Categorías de trabajadores de la salud

Figura 3. Categoría de los asistentes a los cursos de teledermatología en 2016-2017.

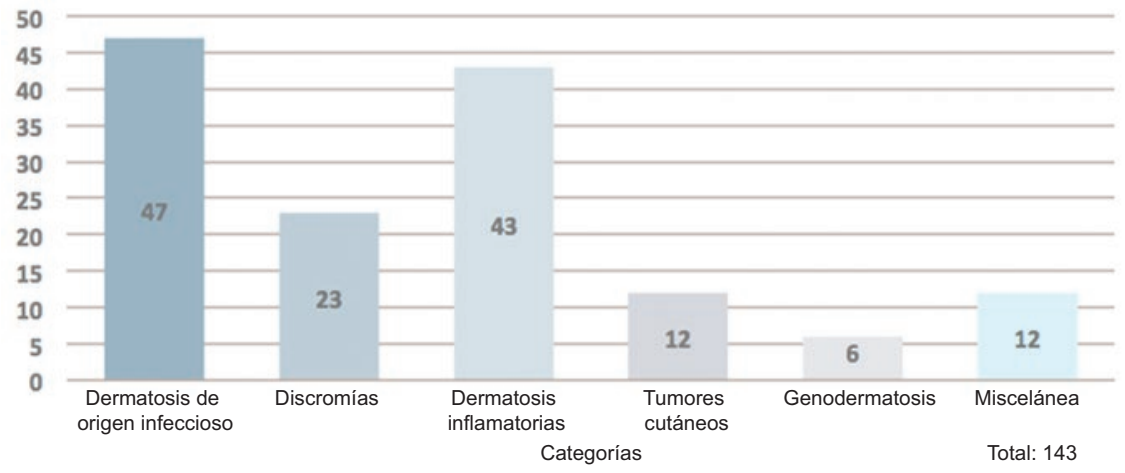

Figura 4. Teleconsultas generadas por personal capacitado en 2016 y 2017 según grupos diagnósticos.

dificultad en su diagnóstico y tratamiento, después de haber sido teleconsultados se canalizan oportunamente al segundo o tercer nivel de atención.

Es importante señalar que el número de teleconsultas se ha incrementado exponencialmente desde el principio de los cursos de teledermatología: iniciamos con 31 pacientes, que aumentaron significativamente a 143 en el segundo año.

La DC ha ido adquiriendo mayor relevancia, como se evidencia en la formación de nuevos grupos dedicados a esta labor con un enfoque diferente, al interactuar con los pacientes, como reportan Casas, et al. ${ }^{14}$ en la Patagonia argentina.

La dermatología continúa teniendo una gran demanda dentro de los servicios de salud, lo que no ha cambiado desde que Bechelli, et al. ${ }^{15}$ señalaron en 1981 que en los países en vías de desarrollo las enfermedades de la piel pueden tener un porcentaje superior al $50 \%$ de la consulta general.

Estrada, et $a l .{ }^{16}$ han señalado el papel que la DC puede tener en la detección y el control de algunas patologías prevalentes en las áreas marginadas, por lo que sería deseable que estos programas continúen abriendo nuevas áreas de desarrollo.

Consideramos que realizar este tipo de trabajo comunitario es una actividad que se ocupa de las clases desprotegidas. Al no poder dar la asistencia presencial hemos tenido que utilizar los recursos tecnológicos disponibles, y demostramos en este trabajo que ha sido posible cubrir los aspectos de asistencia y enseñanza en beneficio de este grupo poblacional.

\section{Bibliografía}

1. Estrada R. Manual de dermatología básica para el personal de salud en el primer nivel de atención. Acapulco, Gro.: Fotopress; 2006.

2. Estrada R, Torres B, Alarcón H, Villegas AA, Martínez E, Chávez-López G, et al. Epidemiología cutánea en dos sectores de atención médica en Guerrero, México. Dermatol Rev Mex. 1992;36:29-34.

3. Estrada R, Romero M, Chávez G, Estrada G. Dermatología comunitaria: diez años de experiencia. Estudio epidemiológico comparativo entre población urbana y rural en el Estado de Guerrero. Dermatol Rev Mex. 2000;44:268-73.

4. Hay R, Andersson N, Estrada R. Community dermatology in Guerrero, México. Lancet. 1991;337:906-7.

5. Andersson N, Martínez E, Villegas A, Rodríguez A. Vigilancia epidemiológica y planificación descentralizada: el uso de sitios centinela en Guerrero. Salud Pub Mex. 1989;31:493-502.

6. Hay, R., S. E. Bendeck, S. Chen, R. Estrada, A. Haddix, T.McLeod, and A. Mahé. 2006. "Skin Diseases." In Disease Control Priorities in Developing Countries, 2nd ed., ed. D. T. Jamison, J. G. Breman, A. R. Measham, 
G. Alleyne, M. Claeson, D. B. Evans, P. Jha, A. Mills, and P. Musgrove, table 37.7. New York: Oxford University Press. (Washington (DC) World Bank; 2006.

7. Hay JR, Estrada R, Grossman H. Managing skin disease in resource-poor environments - the role of community oriented training and control programs. Int J Dermatol. 2011;50:558-63.

8. Estrada CR, Chávez-López MG, Estrada-Chávez G, Paredes-Solís S Specialized dermatological care for marginalized populations and education at primary care level: is community dermatology a feasible proposal? Int J Dermatol. 2012;51:1345-50.

9. Figueroa JL, Fuller LC, Abraha A, Hay R. Dermatology in southwestern Ethiopia: rationale for a community approach. Int J Dermatol. 1998 37:752-8.

10. Finlay AY. Quality of life measurement in dermatology: a practical guide. Br J Dermatol. 1997:135:955-8.

11. Levin YS, Warshaw EM. Teledermatology: a review of reliability and accuracy of the diagnosis and management. Dermatol Clin. 2009; 27:163-76.
12. Bryld LE, Heydenheim M, Dam TM, Dufour N, Vang E, Agner T, et al. Teledermatology with an integrated nurse-led clinic on the Faroe Islands - seven years' experience. J Eur Acad Dermatol Venereol Eur 2011;25:987-90.

13. Lepe V, Moncada B, Castanedo-Cazares JP, Martínez-Rodríguez A, Mercado-Ceja SM, Gordillo-Moscoso A. Primer estudio de teledermatología en México. Una nueva herramienta de salud pública. Gac Med Mex. 2004;140:23-6.

14. Casas IMP, Maino OF, Lupo LR, Merino MF. Implementación de programas de dermatología comunitaria como una manera atractiva de aprender, enseñar y ejercer la especialidad. Rev Med Htal Esp MZA. 20112012;14:22-6.

15. Bechelli LM, Haddad N, Pimenta WPJ, Pangnano PMG, Melchior E Jr, Fregnan RC, et al. Epidemiological survey of skin diseases in school children living in the Purus valley. Dermatologica. 1981;163:78-93.

16. Estrada R, Andersson N, Hay R. Community dermatology and the management of skin diseases in developing countries. Tropical Doctor. 1992;(Suppl 1):3-6. 